\title{
L-MRC Receiver with Estimation Error over Hoyt Fading Channels
}

\author{
Bhagarbjyoti Saikia $^{\# 1}$, Rupaban Subadar*2 \\ ${ }^{\# 1,{ }^{2}}$ Department of Electronics and Communication Engineering, \\ North-Eastern Hill University, Shillong, INDIA. \\ \#1 bhargab.2008@gmail.com \\ *2 rupaban.subadar@gmail.com
}

\begin{abstract}
We present the performance of arbitrary branch maximal ratio combining (MRC) over Hoyt fading channel with estimation error. Among the diversity receiver MRC performs optimally provided the phase and SNR estimation are perfect. Yet, in real life the perfect channel estimation is a challenging job. There may be error present in both phase and the envelope estimation, and the amount of error will be depend upon channel conditions as well as the techniques used for the detection. In this paper performance degradation of the MRC receiver by phase and envelop estimation error have been analyzed in terms of ABER and channel capacity. From the analysis, it can resolve that the receiver operation is affected severely by the estimation error in the envelop than the phase.
\end{abstract}

Keywords-Imperfect channel estimation, Error estimation, MRC, Hoyt Fading, ABER, Channel capacity.

\section{INTRODUCTION}

In the presence of fading, for better performance in wireless communication, different diversity techniques are employed. Among these known techniques the Maximal Ratio Combining (MRC) is the optimal one [1], when there is a perfect estimation of channel on the receiver. But ideally perfect channel estimation is not possible and so in the mathematical modeling imperfect channel estimation (ICE) need to be considered. On the other hand, the Hoyt distribution [2], also referred to as the Nakagami-q distribution, is commonly observed in satellite links subject to strong ionospheric scintillation and in the heavily shadowed environment. Hoyt distribution is frequently utilized to model fading channels severe than Rayleigh. Performance of diversity receivers over iid fading channels are discussed in many research papers for perfect and imperfect channel estimation. The ABER expression of MRC receiver is given in [3] for arbitrary diversity order. The ABER performance of Hoyt fading channels is discussed in [4] but without diversity. MGF (Moment Generating Function) based approach is employed in [5] to derive the ABER expressions. The effect of ICE on MRC receivers over Rician fading channels is studied in [6]. In [7] an expression is derived for Optimum Power and Rate Adaptation over Rayleigh fading channel considering ICE. In [8] the degradation in the average output SNR in terms of phase error for Different diversity combining technique has been demonstrated. The optimum detection and performance of the EGC (Equal Gain Combiner) receiver over Rayleigh faded channel for partially coherent detection is discussed in [9] and [10] respectively. In [11] the effects of phase error in the performance of EGC receivers over correlated Nakagami-m faded channel, using Tikhonov distribution is given. In [12], [13], [14] BER is analyzed to study the performance of M-QAM over Rayleigh, Ricain and Nakagami-m, respectively. In [15] the effect of ICE on the performance of EGC and generalized selection combining receivers is done for MPSK over independent and identically distributed (iid) fading channels like Rayleigh, Rician, Nakagami-m and Hoyt. In [16] capacity analysis is studied for different adaptive receivers considering perfect CSI. Optimal Diversity Combining based on Linear Estimation is given in [17] for the same fading distribution. ABER performance is analyzed to dual branch equal gain combining receiver over the correlated Hoyt fading channel in [18]. In [19] the average symbol error probability (ASEP) and outage probability of the MRC with ICE and co-channel interference (CCI) is presented over Nakagami-m fading channel. Bit error probability and outage probability are analyzed for an arbitrary EGC receiver with phase estimation error in [20]. In [21] a new generalized expression is derived for the bit error probability of M-ary phase-shift keying (MPSK) under additive white Gaussian noise (AWGN) and fading channels. The capacity analysis for dual branch MIMO system over the Hoyt fading channel is presented in [22] considering a perfect channel estimation. The performance of an arbitrary L-branch maximal ratio combining receiver is analyzed for independent Hoyt fading channels in [23]. In [24] the performance of Maximum ratio Combiner for Nakagami-m fading channels is analyzed for coherent and non-coherent detection of Frequency Shift keying (FSK) and Phase Shift Keying (PSK) modulation. A MGF approach also has been discussed in [25] where the ABER performance is studied over $\kappa-\mu$ fading channel considering ICE. In the literature review, we found that the ABER analysis for a diversity receiver with channel estimation error have mainly been studied only for the homogeneous fading channels. This generates a motive to carry out the analysis for nonhomogeneous fading channels like Hoyt. In this paper ABER and capacity analysis is done for an arbitrary L-branch MRC receiver, considering an ICE over the Hoyt fading channel. A mathematical expression for a PDF of this effective SNR over arbitrary iid L- 
branches MRC receiver is derived, which has been used to obtain the ABER expression for coherent BPSK and the capacity analysis. The paper is organized as follows: In section II the system model considered for analysis is explained. In section III PDF of MRC receiver effective output SNR with ICE is discussed. In IV ABER of MRC receiver over Hoyt Fading with ICE is given. In section V Capacity analysis for a L-MRC receiver is done for ICE. In VI the results and analysis is presented. Conclusions are presented in section VII.

\section{SYSTEM MODEL}

The wireless communication system receiving multipath fading signals in the presence of additive white Gaussian noise (AWGN) is considered over here. To receive multipath signals the receiver is equipped with $L$ antennas and perform MRC to improve the quality of the signals. The channel is considered to be slow and frequency non selective. For the assumed channel the received signal over ' $i$ th ' symbol interval is given as,

$$
r(i)=\alpha(i) d(i)+n(i)
$$

where, $d(i)$ is the transmitted symbol in ' $i^{t h}$, interval, with energy $E_{S}=E\left[|d(i)|^{2}\right]$ and noise vector $n(i)=\left[n_{1}(i)\right.$ ,....., $\left.n_{L}(i)\right]^{T}$, is the complex Gaussian noise having zero mean and two sided power spectral density $2 N_{0}$.In this model $\alpha(i)=\left[\alpha_{1}(i), \ldots . ., \alpha_{L}(i)\right]^{T}$ is the channel co-efficient vector for $L$ branches and its elements are Hoyt distributed. The envelop pdf of a Hoyt distributed receive signal is given by [1],

$$
p(\alpha)=\frac{\left(1+q^{2}\right) \alpha}{q \Omega} e^{-\frac{(1+q)^{2} \alpha^{2}}{4 q^{2} \Omega}} I_{0}\left[\frac{\left(1-q^{4}\right) \alpha^{2}}{4 q^{2} \Omega}\right]
$$

where, $\Omega=E\left(\alpha^{2}\right), q \in[0 ; 1]$ is the Hoyt fading parameters and $I_{0}[$.$] is the modified Bessel function of the$ first kind and zero $^{\text {th }}$ order. If the estimated channel vector at the receiver side is denoted as $\hat{\alpha}(i)=\left[\hat{\alpha}_{1}(i), \ldots \ldots . ., \hat{\alpha}_{L}(i)\right]^{T}$, the channel estimation error at each branch can be considered as, $e_{l}(i)=\hat{\alpha}_{1}(i)-\alpha_{l}(i)$. A versatile model for channel estimation error of any arbitrary linear channel estimation is proposed in $[6]$ as,

$$
\alpha_{f, l}(i)=\rho_{l} \hat{\alpha}_{f, l}(i)+z_{f, l}(i)
$$

where, $l=1,2,3, \ldots . ., L, ' f$ ' is the diffused component and the errors $\left\{z_{f, l}(i)\right\}_{l=1}^{L}$ are the iid equivalent estimation error terms with zero mean and variance $\sigma_{z}^{2}$. On the other hand $\rho_{l}$ is denoted as the normalized estimation correlation coefficient between $\alpha_{l}(i)$ and $\hat{\alpha}_{l}(i)$, and can be written as $\rho_{l}=\left|\rho_{l}\right| e^{j \Delta \theta_{l}}$, where $\Delta \theta_{l}$ is the phase offset of $\rho_{l}$. In case of ICE $\left|\rho_{l}\right|<1$ or $\Delta \theta_{l} \neq 0$ or both may arise, which degrades the receiver performance [6].

\section{A. Effective Output SNR with ICE}

Considering ICE to detect the transmitted symbol $d(i)$ at the MRC receiver we have to take the help of complex decision variable (DV), which is given as, $\tilde{D}=\sum_{l=1}^{L} \hat{\alpha}_{l}(i) r_{l}(i)$ [15]. Applying the half plane decision method [21] the complex DV $\tilde{D}$ will be rotated with a plane angle $\beta$ to obtain a new DV as,

$$
D(\beta)=\Re\left(\tilde{D} e^{-j \beta}\right)
$$

where, $\mathrm{M}$ is the constellation size and $\beta=0$ for BPSK $(\mathrm{M}=2)$. So considering the half plane decision method for a DV D, the effective output SNR of a MRC receiver is given as [6],

$$
\gamma_{I C E}^{M R C}=B(\beta) \sum_{l=1}^{L} \hat{\gamma}_{l}
$$

where, B is a function of $\beta$ and given by, $B(\beta)=\frac{\left|\rho_{l}\right|^{2} \cos ^{2}\left(\Delta \theta_{l}-\beta\right)}{\left[\left(1-\left|\rho_{l}\right|^{2}\right) \bar{\gamma}_{l}+1\right]}, \hat{\gamma}_{l}=\left|\hat{\alpha}_{l}\right|^{2} / N_{0}$. 
It is already described that the principle of MRC is based on the channel estimation at the receiver. Hence, it is advantageous to use the adaptive transmission technique in case of MRC receiver. An adaptive technique very the power and rate of transmission based on the channel condition between the transmitter and receiver which is available to the transmitter through a lossless channel from the receiver [7], [16].

\section{PDF OF MRC RECEIVER EFFECTIVE OUTPUT SNR WITH ICE}

Using the Hoyt fading model given in [22] the square of the Hoyt distribution can be written in terms of square Gaussian distribution as,

$$
\alpha_{l}^{2}=X_{l}^{2}+Y_{l}^{2}
$$

where $X_{l}$ and $Y_{l}$ are independent zero mean Gaussian RVs with variances $\sigma_{x}^{2}$ and $\sigma_{y}^{2}$ respectively. In this representation the Hoyt RV $\alpha_{l}$ has the PDF given in (2) where the fading parameter $q=\frac{\sigma_{y}}{\sigma_{x}}$. For the convenience of presentation, but without loss of generality, we assume $\sigma_{x}^{2}=1$ resulting in $\sigma_{y}^{2}=q^{2}$. This channel model is suitable to obtain the PDF of the output SNR of the MRC receiver. From this model doing some mathematical calculation followed by a random variable transformation a closed-form expression for the SNR PDF of MRC receiver is given as [23],

$$
f_{\gamma}(\gamma)=\left(\frac{1+q^{2}}{2 \bar{\gamma} q}\right)^{L} \frac{e}{\Gamma(L)}^{-\left(\frac{1+q^{2}}{\left.2 \overline{q^{2}}\right)}\right)} \gamma_{1}^{(L-1)} F_{1}\left(\frac{L}{2} ; L ; \frac{1-q^{4}}{2 \bar{\gamma} q^{2}} \gamma\right)
$$

Since $\gamma$ and SNR with ICE $\hat{\gamma}$ is identical for $\left|\rho_{l}\right|=1$, so eq.(7) can be reproduced for $\hat{\gamma}$ as,

$$
\begin{aligned}
& f_{\hat{\gamma}}(\hat{\gamma})=\left(\frac{1+q^{2}}{2 \overline{\hat{\gamma}} q}\right)^{L} \frac{e^{-\left(\frac{1+q^{2}}{2 \bar{\gamma} q^{2}}\right) \hat{\gamma}}}{\Gamma(L)} \hat{\gamma}^{(L-1)} \\
& { }_{1} F_{1}\left(\frac{L}{2} ; L ; \frac{1-q^{4}}{2 \overline{\hat{\gamma}} q^{2}} \hat{\gamma}\right)
\end{aligned}
$$

\section{ABER OF MRC RECEIVE OVER HOYT FADING WITH ICE}

In PDF based approach the ABER can be derived by averaging the PDF of the system SNR as [1],

$$
P_{e}=\int_{0}^{\infty} P(e / \gamma) f_{\gamma}(\gamma) d \gamma
$$

where, $P(e / \gamma)$ is a conditional BER. In our analysis the coherent BPSK modulation has been considered and for this the coherent conditional BER is given by [24],

$$
P(e / \gamma)=Q(\sqrt{2 a \gamma})
$$

where, $\mathrm{a}=1$ for Coherent BPSK.

According to the eq.(5) multiplying eq.(8) with the function 'B' followed by a RV transformation the expression can be expressed as,

$$
\begin{aligned}
& f_{\hat{\gamma}}(\hat{\gamma})=\left(\frac{1+q^{2}}{2 \hat{\hat{\gamma}} q}\right)^{L} \frac{e^{-\left(\frac{1+q^{2}}{2 \bar{\gamma}^{2}}\right)^{B^{-1},}}}{B \Gamma(L)} B^{-(L-1)}(\hat{\gamma})^{(L-1)} \\
& { }_{1} F_{1}\left(\frac{L}{2} ; L ; \frac{1-q^{4}}{2 \overline{\hat{\gamma}} q^{2}} B^{-1} \hat{\gamma}\right)
\end{aligned}
$$

substituting eq.(11) and eq.(10) for $\hat{\gamma}$ in eq.(9) and solving the integral using [ [26],6.455(1)], the final ABER expression can be written as, 


$$
\begin{aligned}
& P_{e}=\frac{\frac{1}{2 \sqrt{\pi}}\left(\frac{1+q^{2}}{2 \overline{\hat{\gamma}} q}\right)^{L}}{\Gamma(L) B^{L}} \sum_{k=0}^{\infty} \frac{\left(\frac{L}{2}\right)_{k}}{(L)_{k} k !}\left(\frac{1-q^{4}}{2 B \overline{\hat{\gamma}} q^{2}}\right)^{k} \\
& \frac{\sqrt{a} \Gamma(L+k+0.5)}{(L+k)}\left(\frac{2 B \overline{\hat{\gamma}} q^{2}}{2 a B \overline{\hat{\gamma}} q^{2}+1+q^{2}}\right)^{L+k+0.5} \\
& { }_{2} F_{1}\left(1, L+k+0.5, L+k+1, \frac{1+q^{2}}{2 a B \overline{\hat{\gamma}} q^{2}+1+q^{2}}\right)
\end{aligned}
$$

where, ${ }_{2} F_{1}$ is a Hypergeometric function.

\section{CAPACITY ANALYSIS OF ADAPTIVE MRC RECEIVER WITH ICE}

The adaptive transmission scheme (both power and rate) is employed to increase the receiver performance. In this section we have presented the upper limit of the channel capacities considering the adaptive transmission schemes along with estimation error. The effect of estimation errors on the channel capacities has been discussed in details in the results and discussion section.

\section{A. Optimum Rate Adaptation (ORA):}

Optimal rate adaptation is the simplest adaptive transmission techniques. This method have lot of more practical value due to its simplicity. In this technique adaptation is implemented to the rate of transmission depending on the channel condition while transmitter power remains constant [16],

$$
C_{\text {ora }}=s \int_{0}^{\infty} \log _{2}(1+\gamma) f_{\gamma}(\gamma) d(\gamma)
$$

where, 's' is the bandwidth of the channel. Substituting (8) in (13), calculating for the SNR $\hat{\gamma}$, the equation can be rewrite as,

$$
\begin{aligned}
& \frac{C_{\text {ora }}}{S}=\log _{2} e\left(\frac{1+q^{2}}{2 \overline{\hat{\gamma}} q}\right)^{L} \frac{B^{-L}}{\Gamma(L)} \int_{0}^{\infty} e^{-\left(\frac{1+q^{2}}{2 \bar{\gamma}^{2}}\right)^{-1 / \hat{\gamma}}} \ln (1+\hat{\gamma}) \\
& (\hat{\gamma})^{(L-1)}{ }_{1} F_{1}\left(\frac{L}{2} ; L ; \frac{1-q^{4}}{2 \overline{\hat{\gamma}} q^{2}} B^{-1} \hat{\gamma}\right) d(\hat{\gamma})
\end{aligned}
$$

where, $\frac{C_{\text {ora }}}{s}$ the spectral efficiency $(\mathrm{Bit} / \mathrm{sec} / \mathrm{Hz})$ and ${ }_{1} F_{1}$ is a Hypergeometric function. Solving (14) using $I_{n}(\mu)=\int_{0}^{\infty} t^{n-1} \ln (1+t) e^{-\mu t} d t=(n-1) ! e^{\mu} \sum_{t=0}^{n-1} \frac{\Gamma(-n+t, \mu)}{\mu^{t}}$ the spectral efficiency can be expressed as,

$$
\begin{aligned}
& \frac{C_{\text {ora }}}{s}=\log _{2} e \sum_{k=0}^{\infty} \sum_{t=0}^{(L+k)-1} \frac{\left(\frac{L}{2}\right)_{k}}{\Gamma(L) L_{k} k !}\left(\frac{1-q^{4}}{2 \overline{\hat{\gamma}} q^{2}} B^{-1}\right)^{k} B^{-L}\left(\frac{1+q^{2}}{2 \hat{\hat{\gamma}} q}\right)^{L} \\
& (L+k-1) ! e^{\left(\frac{1+q^{2}}{2 \bar{\gamma} q^{2}}\right) B^{-1}}\left(\left(\frac{1+q^{2}}{2 \overline{\hat{\gamma}} q^{2}}\right) B^{-1}\right)^{-t} \Gamma\left(-(L+k)+t,\left(\left(\frac{1+q^{2}}{2 \overline{\hat{\gamma}} q^{2}}\right) B^{-1}\right)\right)
\end{aligned}
$$

\section{B. Channel inversion with Fixed Rate (CIFR):}

In case of CIFR, the transmitter adapts its power to maintain constant received SNR, so that the inversion of the channel fading effect is possible. Then the channel appears to the encoder and decoder as a time-invariant AWGN channel. The Channel capacity for CIFR is given by [16],

$$
C_{\text {cifr }}=s \log _{2}\left(1+\frac{1}{R_{\text {cifr }}}\right)
$$

where,

$$
R_{c i f r}=\int_{0}^{\infty} \frac{1}{\gamma} f_{\gamma}(\gamma) d(\gamma)
$$


Putting (8) in (17), calculating for the SNR $\hat{\gamma}$ the equation can be written as,

$$
\begin{aligned}
& R_{c i f r}=\sum_{k=0}^{\infty}\left(\frac{1+q^{2}}{2 \overline{\hat{\gamma}} q}\right)^{L} \frac{B^{-L}}{\Gamma(L)} \frac{\left(\frac{L}{2}\right)_{k}}{L_{k}} \frac{\left(\frac{1-q^{4}}{2 B \hat{\gamma} q^{2}}\right)^{k}}{k !} \\
& \int_{0}^{\infty} \hat{\gamma}^{(L+k-2)} e^{-\left(\frac{1+q^{2}}{\left.2 \bar{q}^{2}\right)^{2}}\right)^{-1 / \hat{\gamma}}} d(\hat{\gamma})
\end{aligned}
$$

Solving (18), by using [ [26],351(2)] the expression for $R_{\text {cifr }}$ can be written as,

$$
\begin{aligned}
& R_{c i f r}=\sum_{k=0}^{\infty}\left(\frac{1+q^{2}}{2 \overline{\hat{\gamma}} q}\right)^{L}\left(\frac{1+q^{2}}{2 B \overline{\hat{\gamma}} q^{2}}\right)^{(1-L-k)} \\
& \frac{\left(\frac{L}{2}\right)_{k}\left(\frac{1-q^{4}}{2 B \overline{\hat{\gamma}} q^{2}}\right)^{k} B^{-L}}{L_{k} \Gamma(L) k !}(L+k-2) !
\end{aligned}
$$

substituting, (19) in (16) the final expression for spectral density of CIFR can be obtain.

C. Truncated Channel inversion with Fixed Rate (TIFR):

The major drawback of the CIFR system arises when the channel goes to deep fades. Under this situation, it needs very large amount of power to maintain constant SNR at the receiver. Hence, system may not be practically possible. This leads to a modified version of it with a modification that the transmission suspended if the SNR goes below a threshold value $\gamma_{0}$. The channel capacity of this scheme can be obtained as [16],

$$
C_{\text {tifr }}=s \log _{2}\left(1+\frac{1}{R_{\text {tifr }}}\right)\left(1-P_{\text {out }}\left(\gamma_{0}\right)\right)
$$

where,

$$
R_{\text {tifr }}=\int_{\gamma_{0}}^{\infty}\left(\frac{1}{\gamma}\right) f_{\gamma}(\gamma) d(\gamma)
$$

and

$$
P_{\text {out }}\left(\gamma_{0}\right)=\int_{0}^{\gamma_{0}} f_{\gamma}(\gamma) d(\gamma)
$$

Putting, (8) in (21) and solving the equation, using [26,351(2)], the expression for $R_{\text {tifr }}$ can be expressed as,

$$
\begin{aligned}
& R_{t i f r}=\sum_{k=0}^{\infty}\left(\frac{1+q^{2}}{2 \overline{\hat{\gamma}} q}\right)^{L}\left(\frac{1+q^{2}}{2 B \overline{\hat{\gamma}} q^{2}}\right)^{(1-L-k)} \\
& \frac{\left(\frac{L}{2}\right)_{k}\left(\frac{1-q^{4}}{2 B \overline{\hat{\gamma}} q^{2}}\right)^{k} B^{-L}}{L_{k} \Gamma(L) k !} \Gamma\left[(L+k-1),(L+k-2) \gamma_{0}\right]
\end{aligned}
$$

solving the equation for $P_{\text {out }}\left(\gamma_{0}\right)$, the expression can be obtain as,

$$
\begin{aligned}
& P_{\text {out }}=1-\sum_{k=0}^{\infty}\left(\frac{1+q^{2}}{2 \hat{\hat{\gamma}} q}\right)^{L} \frac{B^{-L}\left(\frac{L}{2}\right)_{k}}{\Gamma(L) L_{k} k !}\left(\frac{1-q^{4}}{2 B \hat{\hat{\gamma}} q^{2}}\right)^{k} \\
& \left(\frac{1+q^{2}}{2 B \hat{\hat{\gamma}} q^{2}}\right)^{-L} \Gamma\left[(L),\left(\frac{1+q^{2}}{2 B \hat{\hat{\gamma}} q^{2}}\right) \gamma_{0}\right]
\end{aligned}
$$

putting (23) and (24) in (20) the final expression for TIFR over Hoyt fading channel for ICE can be obtain. 


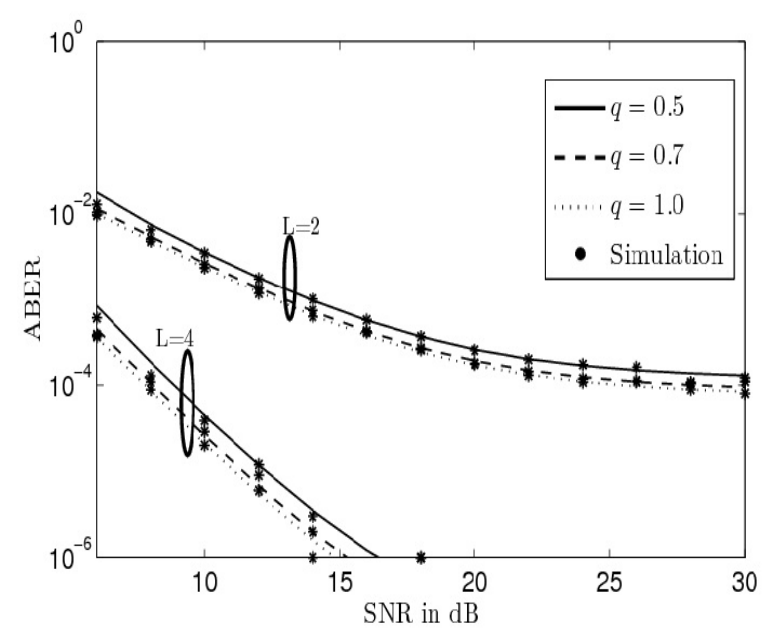

Fig.1: ABER analysis over imperfect Hoyt Fading Channel

$$
\text { for } \rho=0.999, \Delta \theta=\pi / 32
$$

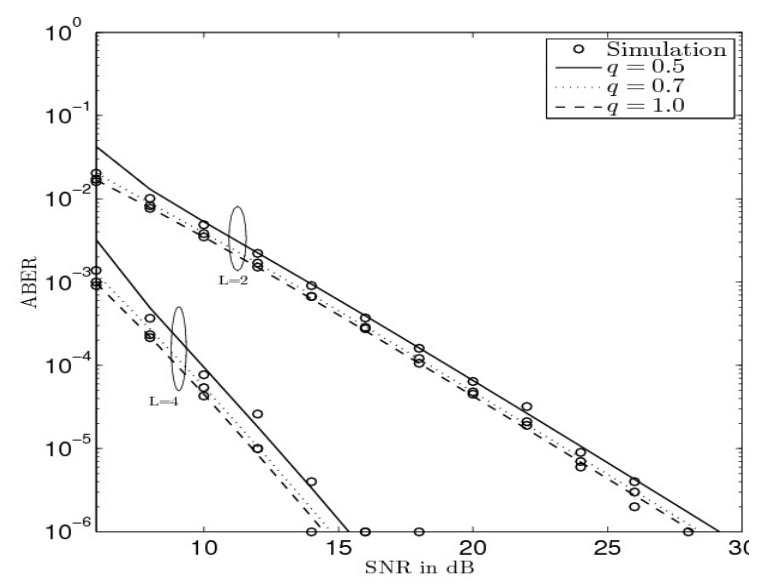

Fig.3: ABER analysis over imperfect Hoyt Fading Channel

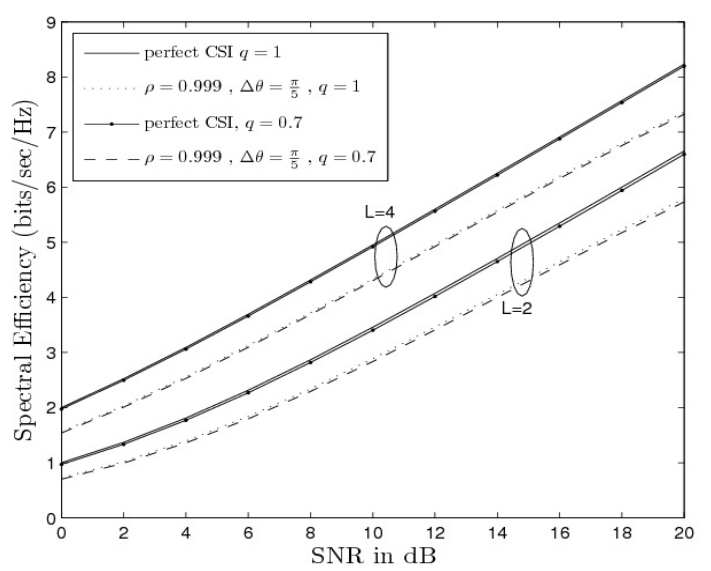

Fig 5: Comparison of Spectral Efficiency considering ICE, with perfect CSI in CIFR

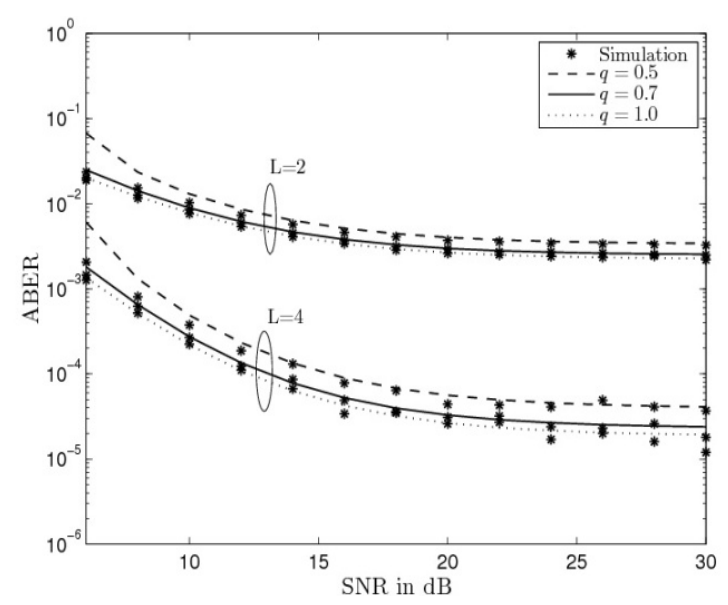

Fig. 2: ABER analysis over imperfect Hoyt Fading

Channel for $\rho=0.995, \Delta \theta=\pi / 20$

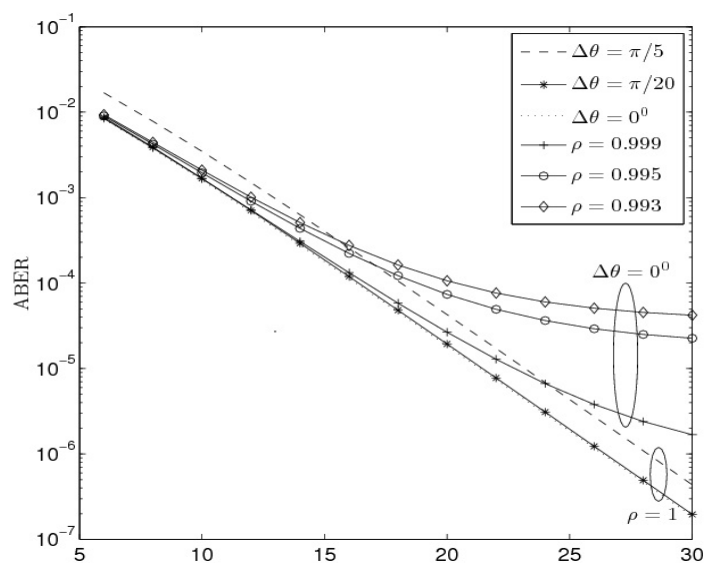

Fig.4: ABER comparison with perfect CSI over

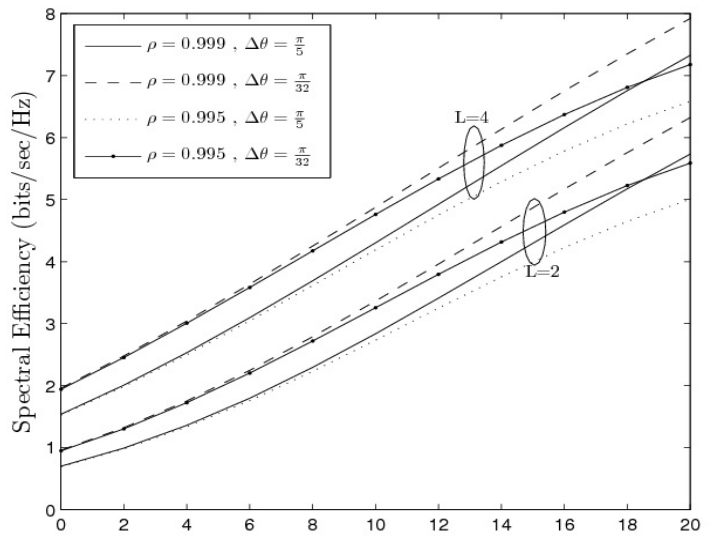

Fig 6: Effect of $\rho$ and $\Delta \theta$ on Spectral

Efficiency in CIFR ( Where $q=0.7$ ) 


\section{RESULTS AND DISCUSSION}

The analytical results of the above expression of an MRC system, has been presented in this section with proper analysis. ABER vs $\bar{\gamma}$ has been shown for various channel conditions (for different fading parameter ' $\mathrm{q}$ '), diversity order ( $\mathrm{L}$ ), phase and envelope estimation errors from Fig. 1 to Fig. 4 . We have observed similar observation presented in the earlier literature for parameters ' $\mathrm{L}$ ' and ' $\mathrm{q}$ ' when there is no estimation error. However, the performance completely changes with the amount of estimation error. In Fig.1 the amount of envelope estimation error ' $\rho$ ' is considered as 0.999 and the phase $\Delta \theta=\pi / 32$. As expected with the decrease of ' $q$ ' parameters of Hoyt distribution the ABER is increasing. But if we increase the antenna number at the receiver side (for $\mathrm{L}=4$ ) the BER decrease considerably. The same thing can be seen in Fig. 2 where, $\rho=0.995$ and $\Delta \theta=\pi / 20$ has been considered. But comparing both the cases it is clear that, since in Fig. 2 more estimation error has been taken; so the ABER is remarkably high in this case (Ex: For $\mathrm{L}=2$, when SNR is $20 \mathrm{~dB}$, ABER is $10^{-2.1} \mathrm{bit} / \mathrm{sec}$ ) than the first case (Ex. For $\mathrm{L}=2$, when SNR is $20 \mathrm{~dB}$, ABER is $10^{-3.9} \mathrm{bit} / \mathrm{sec}$ ). In Fig. 3, we have considered only the phase error and have found that, ABER is comparatively low than the first two cases (Ex. For $\mathrm{L}=2$, when SNR is $20 \mathrm{~dB}$, ABER is $10^{-4.6} \mathrm{bit} / \mathrm{sec}$ ). From this we can conclude that the accuracy in the estimation of envelope is much more essential than phase for an MRC receiver. A comparative study of perfect CSI with ICE has been shown in Fig. 4. From the figure, if $\Delta \theta=0$ (No phase error) but enveloped error is there, then at $30 \mathrm{~dB}$ SNR, ABER is $10^{-5.6} \mathrm{bit} / \mathrm{sec}$, with a slight change in the envelope error " $\rho^{\prime}$, from 0.999 to 0.995 , ABER increase considerably $10^{-4.4} \mathrm{bit} / \mathrm{sec}$ ). Then the performance analysis is shown in terms of channel capacity from Fig. 5 to Fig. 6 for an adaptive transmission system. In Fig.5 a comparison of channel capacity (in terms of spectral efficiency) has been given for perfect vs imperfect estimation considering CIFR system. The analysis has been done for two different ' $q$ ' values (for $q=1$ and $q=0.7$ ) considering $\rho=0.999$ and $\Delta \theta=\pi / 5$. From here it is clear that, the channel capacity is reduced remarkably for imperfect estimation irrespective of the diversity order. In Fig: 6 the effect of ' $\rho$ ' (Envelop error ) and $\Delta \theta$ (Phase error ) on channel capacity is studied. As expected for channel capacity also the effect of enveloped error is comparatively more sensitive than the phase error irrespective of order of diversity for a MRC system.

\section{VII.CONCLUSION}

This paper analyzes the performance of L-MRC receiver with estimation error over Hoyt fading channels. The expression for ABER and capacity has been derived considering both envelope and phase estimation error. Form the analysis it can be concluded that the performance of MRC receiver degrades considerably with improper estimation. Infect, from the result we found that if the envelope estimator is not accurate enough it is better not go for the MRC receiver. Inaccurate estimator may completely nullify the advantages expected from the MRC system.

\section{REFERENCES}

[1] M. K. Simon and M.S. Alouini, "Digital Communication over Fading Channels" John Wiley and Sons Inc., 2005.

[2] R.S. Hoyt, "Probability functions for the modulus and angle of the normal complex variate" Bell. Syst. Tech. J., vol. 26, pp. 318-359, April 1947.

[3] I. Korn and J. P. Fonseka, "M-CPM with MRC diversity in Rician-, Hoyt-, and Nakagami-fading channels" IEEE Trans. of Veh. Tech., vol.50, no. 4, pp. 1182-1189, July 2001.

[4] R. M. Radaydeh, "Average error performance of M-ary modulation schemes in Nakagami-q Hoyt fading channels" IEEE Commun. Lett., vol. 11, no. 3, pp. 255-257, March 2007.

[5] T. Q. Duong, H. Shin, and E. -K. Hong "Error probability of binary and M-ary signals with spatial diversity in Nakagami-q (Hoyt) fading channels" Eurasip Journal on Wireless Commun. and Networking, vol. 4, pp. 1-8, October 2007.

[6] Y. Ma, R Schober and S Pasupathy, "Effect of channel estimation error on MRC Diversity in Rician Fading Channels" IEEE Trans. on Vehicular Tech., vol. 54,pp. 2137-2142, November 2005.

[7] Ali Olfat and Mahammad Shikh Bahaei, " Optimum power and rate adaptation with Imperfect Channel Estimation for MQAM in Rayleigh Flate Fading Channel" IEEE Transaction and Vehicular Technology, Vol. 57, No 4, pp 2622, July 2008.

[8] D. G. Brennen, "Linear Diversity Combining Techniques" Proceedings of the IRE,vol. 47, pp. 1075 - 1102, June 1959.

[9] M. A. Najib and V. K. Prabhu, "Analysis of Equal-Gain Diversity with Partially Coherent Fading Signals" IEEE Trans. on Vehicular Technology, vol. 49, pp. 783-791, May 2007.

[10] Andrew Viterbi, "Optimum Detection and Signal Selection for Partially Coherent Binary Communication" IEEE Trans. on Information Theory, vol. 11, pp. 239 -246, April 1965.

[11] N. C. Sagias and GeorgeK. Karagiannidis, "Effects of Carrier Phase Error on EGC Receivers in Correlated Nakagami-m Fading" IEEE Commun. Letters, vol. 9, pp. 580-582, July 2005.

[12] Yao Ma, R. Schober and Dongbo Zhang, "Exact BER for M-QAM with MRC and Imperfect Channel Estimation in Rician Fading Channels" IEEE Trans. on Wireless Commun., vol. 6, pp. 926-936, March 2007.

[13] Yao Ma and Jinghua Jin, "Effect of Channel Estimation Errors on M-QAM with MRC and EGC in Nakagami-m fading channels" IEEE Trans. on Vehicular Technology, vol. 56, pp. 1239-1250, May 2007.

[14] Xiaoyi Tang, Mohamed-Slim Aloini and Andrea J. Goldsmith, "Effect of channel estimation error on M- QAM BER performance in rayleigh fading" IEEE Trans. on Commun., vol. 47, pp. 1856-1864, December 1999. 
[15] Y. Ma, R. Schober and S. Pasupathy, "Performance of M-PSK with GSC and EGC with gaussian weighting errors" IEEE Trans. on Vehicular Tech., vol. 54, pp. 149-162, January 2005

[16] A.J. Goldsmith and P. P. Varaiya, "Capacity of Fading Channel With Channel Side Information" IEEE Transaction On Information Theory" Vol, 43,No. 6, pp.1986-1992,November 1997

[17] Jingxian Wu and Chengshan Xiao, "Optimal Diversity Combining based on Linear Estimation of Rician Fading Channels" IEEE Trans. on Commun., vol. 56, pp. 1612-1615, October 2008.

[18] G. Aruna, P.R Sahu, "ABER of EGC receiver over Co-related Hoyt fading channels with phase error and co-channel interference" Annual IEEE India Conference (INDICON),pp 936-940, December 2012.

[19] G. Aruna, P.R Sahu, " Performance analysis of MRC receiver with channel estimation error and CCI in Nakagami-m fading channel" Computational Intelligence and Computing Research (ICCIC), pp 1-4, December 2014.

[20] G Aruna, " PDF methodology of analysis of L-branch Equal Gain Combiner with carrier phase error and CCI over nakagami-m fading" International Conference on Computing and Network Communications (CoCoNet), pp 505-510, December 2015.

[21] Woojin Jeong, Jaeyoon Lee, and Dongweon Yoon, "New BER Expression of MPSK"IEEE Trans. on Vehicular Technology, vol. 60.No 4, pp 1916-1924, May 2011.

[22] G. Fraidenraich, O. L'ev^eque and J. M. Cioffi, "On the MIMO channel capacity for the dual and asymptotic cases over Hoyt channels" IEEE Commun. Lett., vol. 11, No. 1, pp. 31-33, January 2007.

[23] R. Subadar, P.R. Sahu, "Performance of L-MRC receiver over independent Hoyt fading channels"'" NCC Conference, pp 1-5, January 2010.

[24] V. A. Aalo, "Performance of maximal-ratio diversity systems in a correlated Nakagami-fading environment" IEEE Trans. on Commun., vol. 43, No. 8, pp. 2360-2369, August 1995.

[25] Pawan Kumar, Dr. Pravas Ranjan Sahu "ABER Performance of MRC Receiver in $\kappa-\mu$ Fading with Channel Estimation Error" M. Tech Thesis, IIT Guwahati, 2013.

[26] I.S. Gradshteyn and I. M. Ryzhik "Tables of Integrals, Series And Products," Elsevier Inc,2007.

\section{AUTHOR PROFILE}

Bhargabjyoti Saikia completed his engineering degree (M Tech) in Information Technology from North Eastern Regional Institute of Science and Technology of Arunachal, India. Presently he is working as an Assistant Professor at Electronics and Communication Engineering Dept. of Dibrugarh University, Assam, India and associated with the research work at Dept. of Electronics and communication of North Eastern Hill University, Shillong, Maghalaya.

Rupaban Subadar He has completed his Ph.d. in wireless communication from IIT, Guwahati. Now he is working as an Associate Professor in Dept. of Electronics and Communication Engineering of of North Eastern Hill University, Shillong, Maghalaya. His research interest is in wireless communication. 